This PDF is a selection from a published volume from the National Bureau of Economic Research

Volume Title: Discoveries in the Economics of Aging

Volume Author/Editor: David A. Wise, editor

Volume Publisher: University of Chicago Press

Volume ISBN: 0-226-14609-X (cloth); 978-0-226-14609-6 (cloth);

978-0-226-14612-6 (EISBN)

Volume URL: http://www.nber.org/books/wise13-1

Conference Date: May 9-11, 2013

Publication Date: June 2014

Chapter Title: Expectations, Aging, and Cognitive Decline

Chapter Author(s): Gábor Kézdi, Robert J. Willis

Chapter URL: http://www.nber.org/chapters/c12980

Chapter pages in book: (p. 305 - 337) 


\title{
Expectations, Aging, and Cognitive Decline
}

\author{
Gábor Kézdi and Robert J. Willis
}

This study explores the relationships between expectations, aging, and cognitive decline, a topic that has previously received little research. The empirical literature on the individual heterogeneity of expectations is relatively new, and little has been published in this area, especially with respect to the general population. (See Hurd [2009] for a review of the empirical literature.) However, heterogeneity in expectations is likely to play an important role in accounting for heterogeneity in decisions made in the presence of uncertainty. If aging has direct effects on the ways in which people form expectations, then those effects may affect the quality of important decisions made in old age.

In this chapter we use data from the Health and Retirement Study (HRS) to document general patterns in expectations in various domains with respect to aging and investigate the potential role of cognitive decline in those patterns. We focus on two aspects of expectations: optimism and uncertainty. The HRS measures expectations by asking about the probability of various events (see Manski [2004], for the case for probabilistic measurement of expectations using surveys). We define optimism as the assignment of higher

Gábor Kézdi is associate professor of economics at Central European University and a research fellow of the Institute of Economics at the Hungarian Academy of Sciences. Robert J. Willis is professor of economics and research professor in the Department of Economics and the Institute for Social Research at the University of Michigan and a research associate of the National Bureau of Economic Research.

We are grateful to the Behavioral and Social Research Program of the National Institute on Aging (NIA) for supporting through grant U01-AG09740 the collection of the HRS data used in this chapter and for research support through NIA grant P01AG026571. We are also grateful to Péter Hudomiet for excellent research assistance. For acknowledgments, sources of research support, and disclosure of the authors' material financial relationships, if any, please see http://www.nber.org/chapters/c12980.ack. 
probabilities to events with positive consequences. We define uncertainty as a person's inability or unwillingness to state a probability belief or evidence of ambiguity or vagueness in the beliefs that they do report. Our measure of uncertainty is the propensity to answer "don't know" or "50 percent." (Hudomiet and Willis [2013] use a similar concept of uncertainty but measure uncertainty in a different way.) Both optimism and uncertainty should be important for decision making: optimism can have direct effects by shifting the level of expectations, while uncertainty can affect decisions through interactions with risk aversion or through more subtle preferences such as ambiguity aversion or loss aversion.

Our major aim in this chapter is to provide descriptive evidence using longitudinal data from the HRS without imposing much theoretical structure or seeking causal results. We do, however, pay close attention to methodological issues involving measurement error, calendar time effects, cohort differences and mortality selection to avoid findings that are statistical artifacts rather than patterns associated with aging. Our treatment of cohort effects and mortality selection builds on the approach of Agarwal et al. (2009), who argue that roughly after age fifty, aging leads to an increase in "mistakes" in decision making that may be due to cognitive decline. Our treatment of calendar time effects makes use of variation in wave-to-wave changes in age induced by variation of the timing of interviews within a survey wave. We document substantial measurement error in cognitive decline and discuss its consequences for our joint analysis of cognitive decline and expectations. We minimize the consequences of measurement error by determining the individual rates of change in the variables across all the survey waves rather than analyzing wave-to-wave changes. We perform several robustness checks to substantiate the resulting associations.

Although both optimism and uncertainty can be specific to the events in question, we show empirically that aging may have general effects on both optimism and uncertainty. In most cases, aging appears to decrease optimism and increase uncertainty. Optimism with respect to stock market expectations, expectations that income will keep up with inflation, and expectations of sunshine the next day all decline strongly with age. The increase in uncertainty is less robust and depends on the measure of uncertainty. Survival expectations are an exception, with a significant increase in optimism and potentially a decrease in uncertainty observed with age. Increasing optimism about survival was documented earlier by Hurd, Rohwedder, and Winter, (2005) for the European countries in SHARE and by Hudomiet and Willis (2013) using data from the HRS with a different measurement strategy.

Aging could have these general effects for several reasons. One possibility is that cognitive decline associated with aging affects an individual's view of the world and their ability to process information about the world, causing a person to overstate the likelihood of negative events and to hold less precise probabilistic beliefs. Our results provide some support for this possibility, as 
we find that cognitive decline plays a modest but statistically significant and robust role in explaining the decline of optimism with the exception of survival expectations. Somewhat surprisingly, we do not find an association between cognitive decline and increasing uncertainty.

Another possibility is that the increase in the awareness of mortality that accompanies aging leads to decreased attention to events that are far in the future, thus reducing incentives to acquire knowledge about those events. ${ }^{1}$ As an individual's economic focus shifts from work to retirement and from the accumulation of wealth to managing that wealth during retirement, the relevance of particular types of economic events may change. In the same way, from a psychological point of view, Carstensen (2006) theorizes that aging makes people focus less on long-term goals and more on nearterm emotional sources of satisfaction. These economic and psychological dimensions of an individual's changing perspective of time suggest that aging may lead to reduced attention to macroeconomic events. Our results on the differential effects of age and cognitive decline on survival expectations may be interpreted as support for this theory. However, the tendency for aging and cognitive decline to reduce optimism in most of the domains we investigate seems contrary to Carstensen's theory.

We begin our analysis by deriving simple measures of optimism and uncertainty about particular topics from HRS questions about subjective probability beliefs regarding stock market returns one year in the future, the chance of a future economic depression, whether tomorrow will be a sunny day, whether one's income will keep up with inflation, job loss, and survival to a specific age. ${ }^{2}$ Next, we show how these measures change with age, employing methods to isolate "pure" age effects by eliminating cohort and time effects. We then turn our attention to measures of cognition from the HRS and describe the process of cognitive decline with age. We provide evidence of substantial survey noise in the cognitive measures and discuss the implications of this noise for our analysis. Finally, we examine how changes in optimism and uncertainty in each domain are related to cognitive decline using techniques that minimize the potential of spurious relationships.

\subsection{Data}

This study uses data from seven waves of the Health and Retirement Study (HRS), spanning from 1998 to 2010. The HRS began in 1992 with a cohort of individuals age fifty-one to sixty-one and their spouses. In 1998,

1. See Kézdi and Willis (2012) for a model showing how expectations about stock market returns are affected by incentives to learn about the history of returns and other aspects of financial investment. They also show that greater stock ownership is associated with more optimistic expectations.

2. The sunny day question of the HRS has been used as a measure of optimism by Bassett and Lumsdaine (1999). 
Table 9.1

The expectation questions of the HRS used in this analysis

Question label

Exact wording of the question

Stock market

By next year at this time, what is the percent chance that mutual fund shares invested in blue chip stocks like those in the Dow Jones Industrial Average will be worth more than they are today?

Economic depression What do you think are the chances that the US economy will experience a major depression sometime during the next ten years or so?

Sunny day

What do you think are the chances that it will be sunny tomorrow?

Income growth

What do you think are the chances that your income will keep up with inflation for the next five years?

Job loss

What are the chances that you will lose your job during the next year?

Survival to age $A$

What is the percent chance that you will live to be $A$ or more?

(with $A$ being a function of the age of the respondent)

the sample was refreshed to make it representative of all age groups above fifty years of age. The spouses of all respondents were also interviewed, regardless of their age. The sample has been refreshed with a new six-year cohort of fifty-one to fifty-six-year-olds and their spouses every six years (in 2004 and 2010), and 2010 is the currently the last wave with available data. We use data on all individuals who were interviewed in at least two survey waves and were between fifty-one and ninety years old at the time of each interview. Proxy interviews were discarded because they lack observations on expectations. Altogether, we analyzed 107,024 observations of 20,938 individuals.

The HRS has asked respondents to assess the probability of various outcomes since its beginning in 1992. This analysis focuses on the six questions listed in table 9.1.

Respondents were invited to answer these expectation questions in percentage terms. The question sequence was introduced by explaining the task and providing an example of the chance of rain on the day following the interview. The sunny day question was used as a warm-up question in some survey waves. Not every question was asked in every wave of the HRS: of the six questions we analyze here, only the survival question was asked every time. We display the number of individuals in our sample who were asked each question in each survey wave in table 9.2. Not every expectation question was asked of each respondent, but five of the six questions we analyze were asked of all participants in at least some of the survey waves (the exception is the job loss question, which was restricted to respondents who were employed). Aside from general availability, the main motivation behind selecting these six questions is the fact that it is relatively straightforward to 
assign positive or negative meaning to these questions, which is important for our analysis of optimism.

We analyze two aspects of expectations: optimism and uncertainty. We define optimism as assigning higher probabilities to events that have positive consequences. It is relatively straightforward to operationalize this definition for the probability answers examined here: the measure of optimism is the probability answer itself. We redefined answers to the economic depression and job loss questions by subtracting them from 100 percent so that the resulting percentages also correspond to the positivity of the answer. To handle potential spurious trends in the underlying "true" probabilities, we made two additional adjustments. First, we discarded the sunny day answers of respondents who moved between interviews. This allows us to ensure that age-related changes in residence (e.g., to retirement communities in southern states) do not affect measured changes in responses to the sunshine question. Seasonal changes are accounted for by dummy variables expressing the month of the interview as described later. Second, we replaced the answer to the survival question with the difference between the respondent's answer and the corresponding probability reported by life expectancy tables. $^{3}$

Conceptually, we define uncertainty as a person's inability to form a probabilistic belief or his admission that his beliefs are imprecise. For each expectation question, we measure uncertainty in terms of the propensity to answer "don't know" or "50 percent." "Don't know" clearly signals an inability to form probabilistic expectations. The "50 percent" answers are interpreted in a similar way based on the assumption that most respondents mean "unsure" when they say " 50 percent." This assumption is supported by evidence. Beginning in 2006, the HRS asked a follow-up question to people who answered "50 percent" to the stock market question and the survival expectation question. For example, among respondents who answered " 50 " to the stock market question, a follow-up question asked whether they thought it was equally likely that the market would go up or go down or whether they were "just unsure." Seventy percent of these respondents for both the stock market and survival questions answered that they were unsure. The results are qualitatively the same but quantitatively stronger when we exclude the 50 percent answers and measure uncertainty only by the propensity to answer "don't know."

In addition to establishing the effects of aging, our analysis aims to uncover whether those effects are related to the decline of cognitive functioning. Cog-

3. These implied survival probabilities were compiled from the appropriate life table for each gender, year of age, and survey year. The variable is part of the RAND distribution, which expresses the HRS as the ratio of the answer to the survival probability question divided by the probability implied by the life expectancy tables. We transformed that variable to measure the difference instead of the ratio. The RAND documentation is available at http://www.rand .org/content/dam/rand/www/external/labor/aging/dataprod/randhrsL.pdf, pages 1019-1025. 
nitive functioning is measured by a composite twenty-seven-point variable that combines results from four short cognitive tests that were administered in each wave of the HRS that we use. These four tests were two word recall tests, a counting backward test, and the "serial sevens" test. The first test asked respondents to recall ten words immediately after hearing them from the interviewer, within one minute, while the second test asked respondents to complete the same task some time later after answering other survey questions. These two tests were scored according to the number of correctly recalled words. The third test asked respondents to count backward from 20 , with a score of 1 assigned for a correct answer. The fourth test asked respondents to subtract 7 from 100 , subtract 7 from the result, and so forth for 6 subtractions. The score for this test is the number of correct subtractions. We quantify cognitive functioning with the combined score that has previously been used in the literature investigating cognitive functioning using HRS data. ${ }^{4}$

In some of our analyses, we examine the association with normal cognitive aging as distinct from associations with the onset of dementia. Dementia is a loss of cognitive functioning beyond normal aging. Dementia may cause people to be unable to answer complex survey questions such as the expectation questions. Most severely demented respondents participate in the HRS via proxy interviews, and these respondents are not asked to perform the cognitive tests and answer the expectation questions. Therefore, it is not possible to directly analyze the association between dementia and expectations in these data. At the same time, signs of the onset of dementia can be detected in our sample using the prediction model developed by Hurd et al. (2013). Using a clinical diagnosis of dementia in the ADAMS study of a subset of HRS respondents (Plassman et al. 2007), Hurd et al. (2013) assigned predicted probabilities of dementia status one year after the interview for every respondent in the HRS. These predictions use variables observed in the HRS and combine those variables into probabilities using an ordered probit model with three outcomes (dementia, severe impairment without dementia, and normal aging). For nonproxy interviews, this prediction uses the cognitive score, the change in the cognitive score from the previous interview, demographic characteristics, and measures of assistance with activities of daily living (ADL). The correlation between the decline in the cognitive score and the predicted probability of dementia would make joint analysis of cognitive ability and dementia problematic. The predicted probability of dementia is practically zero below age seventy, which further limits the ability to conduct joint analysis. Instead, we use the predicted probability of dementia in our robustness checks to determine whether asso-

4. A fifth measure is often added to the score to control for dementia (Crimmins et al. 2011), but we use a different, more reliable measure of dementia and do not include that score in our cognitive measure. The HRS cognitive measures are described in more detail in Fisher et al. (2012). 
Number of observations for the expectation questions and cognitive measures by survey wave

\begin{tabular}{lrrrrrrr}
\hline & \multicolumn{7}{c}{ HRS survey wave } \\
\cline { 2 - 8 } Question label & \multicolumn{1}{c}{2098} & 2000 & 2002 & \multicolumn{1}{c}{2004} & 2006 & 2008 & 2010 \\
\hline Stock market & 0 & 0 & 13,412 & 16,647 & 15,874 & 15,045 & 13,491 \\
Economic depression & 3,400 & 108 & 149 & 16,647 & 15,874 & 15,045 & 0 \\
Sunny day & 0 & 14,792 & 15,451 & 16,867 & 0 & 0 & 0 \\
Income growth & 14,591 & 14,792 & 15,451 & 16,867 & 16,266 & 0 & 0 \\
Job loss & 4,847 & 4,506 & 3,943 & 4,925 & 4,393 & 0 & 3,192 \\
Survival to age $A$ & 7,169 & 13,894 & 14,807 & 15,899 & 15,241 & 15,040 & 13,432 \\
Cognitive score & 14,591 & 14,792 & 15,463 & 16,912 & 16,280 & 15,358 & 13,628 \\
Probability of dementia & 0 & 4,299 & 5,005 & 5,320 & 5,572 & 5,802 & 5,662 \\
\hline
\end{tabular}

Source: HRS waves from 1998 through 2010. Sample includes respondents ranging in age from fifty-one to ninety years old, without the new respondents in 2010 and without the proxy interviews. The number of observations refers to the number of individuals in the sample who were asked the relevant question (including individuals who refused to answer or who responded that they did not know).

ciations with cognitive decline correspond to normal aging or early signs of dementia.

The income growth question was discontinued in 2008. The economic depression question was discontinued in 2010, and prior to 2004, it was asked of new respondents only (new spouses and the new cohort in 1998). The stock market question was first asked in 2002. The job loss question was asked in all waves except for 2008, but only of the subset of respondents who were employed at the time of the interview. Survival expectations were asked of the entire sample in all waves except in 1998, when it was only asked of a subsample.

\subsection{Expectations and Aging}

The effect of aging on expectations is difficult to measure for many reasons. Cross-sectional age profiles blend the effect of aging with differences across birth cohort and selective mortality. Cohort differences may lead to cross-sectional age differences in expectations if older birth cohorts have different expectations than younger birth cohorts, even when their answers are compared at the same age. Selective mortality may lead to cross-sectional age differences in expectations if mortality is correlated with expectations (perhaps due to common factors).

Examining changes in expectations for the same individuals eliminates confounding cohort effects. Age profiles can be constructed from the individual changes by creating aggregate slopes and combining those slopes (this method was used by Agarwal et al. [2009]). The slopes of the average measures are defined as 


$$
s(x)_{a}=\frac{1}{N_{\Omega(a)}} \sum_{i \in \Omega(a)} \frac{x_{i, w+1}-x_{i, w}}{a g e_{i, w+1}-a g e_{i, w}},
$$

where $x$ is the relevant variable, $s(x)_{a}$ is the slope starting with integer age $a$, $i$ refers to individuals, $w$ refers to the survey wave, age in the denominator is measured in fine detail (in 1/12th years, calculated from the month of birth of the individual to the month of the interview), and $\Omega(a)$ refers to the set of individuals belonging to an age group defined by integer age $a$. Once the slopes are estimated, they can be added from a prespecified starting value to create age profiles identified from wave-to-wave changes.

However, wave-to-wave changes blend the effects of aging with the effects of calendar time. Calendar time may affect most of the expectations measured here, including those regarding income growth, economic depression, the stock market, or the probability of a sunny day.

Fortunately, the features of the data collection help us to control for calendar time effects. The data collection of any survey is spread out over time. In a typical HRS wave, over 80 percent of the interviews are completed within six months, and the remaining interviews take another five to eight months to collect. This leads to interindividual variation in the time that passes between interviews. Measured to monthly precision, the median amount of time between two interviews is exactly two years, the 1st decile is 1 and 9/12 years, the 9 th decile is 2 and 6/12 years, and the tails are long. As a result, the wave-to-wave difference in any measure may be related to different age differences between waves for different individuals.

We control for calendar time effects by replacing each expectation variable with its deviation from the mean measured in the year-month of the interview. That is, we replace variable $x$ in equation (1) by the following variable:

$$
\tilde{x}_{i w}=x_{i w}-\frac{1}{n_{m}} \sum_{j \in \Omega(m)} x_{j w},
$$

where $m$ refers to the year-month of the interview and $\Omega(m)$ refers to the set of all observations in our sample in year-month $m$. Identifying the age slopes from the year-month adjusted variables uses the assumption that calendar time has an equal effect for all respondents. ${ }^{5}$ Under that assumption, the age

5. The age slopes of the year-month adjusted variables are identified from differences-indifferences-in-differences. Consider two respondents of exactly the same age interviewed in the same month in the base wave. One respondent is interviewed in exactly two years in the following wave, by which time her age increased by two years. The other respondent is interviewed six months later, so that his age increased by 2.5 years. The difference in the changes of their answers may reflect differences in aging or differences in the calendar time of the second wave. If we assume that the differences resulting from the difference in calendar time are the same for all respondents, we can estimate that difference using pairs of respondents with the same calendar time difference between their second interviews and the same time elapsed from their base interview to their second interview. If there is a difference in this second comparison, we record that difference and subtract it from the difference measured in the first comparison. 
profiles constructed using age slopes of the year-month adjusted variables $s(\tilde{x})_{a}$ show the effects of age without cohort effects and without calendar time effects.

\subsubsection{Age Profiles of Optimism}

We first show the age profiles of optimism by displaying expectations as a function of age. Recall that we adjusted some of the expectation variables to reflect cleaner measures of optimism than the original answers. First, we inverted the answers to the economic depression and job loss questions so that higher values reflect more optimistic expectations. Second, we discarded the sunny day answers of respondents who had moved to another location since their previous interview. Third, we replaced the answer to the question about survival to age $A$ with the difference between the survey answer and the probability obtained from life expectancy tables. All answers were replaced by their deviations from their year-month average.

Figure 9.1 presents the results. In each graph, the dashed line represents the cross-sectional age profile of the original answers, while the solid line reports the age profile constructed from the cumulative slopes of the yearmonth adjusted answers. The figures show the bootstrap 95 percent confidence intervals around the curves, colored as lighter gray for the cumulative slopes and darker gray for the cross-sectional profiles. ${ }^{6}$ With the exception of the survival probability answers, which will be discussed in more detail later, the cross-sectional age profiles blend cohort, time, and selection effects with age effects, while the age profiles from the cumulative year-month adjusted slopes show pure age effects. Each graph includes a horizontal line at the level of the optimism measured at age fifty-one, the normalized starting point for the age profiles.

Age has a negative effect on optimism in five of the six cases, and this effect is statistically significant in the case of the stock market, sunshine, and real income growth expectations. While specific explanations can be constructed for some of the figures (aging may lead to lower real income), it is harder to do so for other figures (the stock market or the sunny day). Therefore, there may be a general negative effect of age on optimism in the domains represented by the three figures.

The solid-line profiles based on cumulative slopes can be thought of as robustness checks for the dashed cross-sectional profiles that remove the potential effects of birth cohort and selection. With the exception of survival expectations, the solid lines are not statistically significantly different from the dashed lines. Most importantly, whenever the dashed cross-sectional

6. The boostrap procedure involved constructing entire histories of answers of households (spouses together) and repeating the entire estimation procedure within each bootstrap draw. We expect confidence intervals to be wider for the profiles of cumulative age-adjusted differences because the role of measurement error and other time-varying idiosyncratic variations in the answers is magnified by taking differences. 


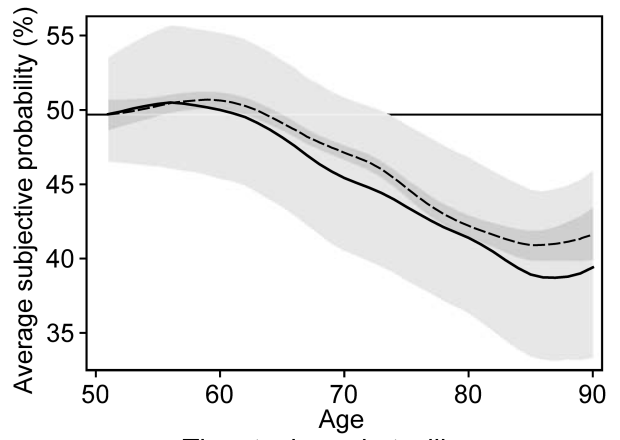

a. The stock market will go up

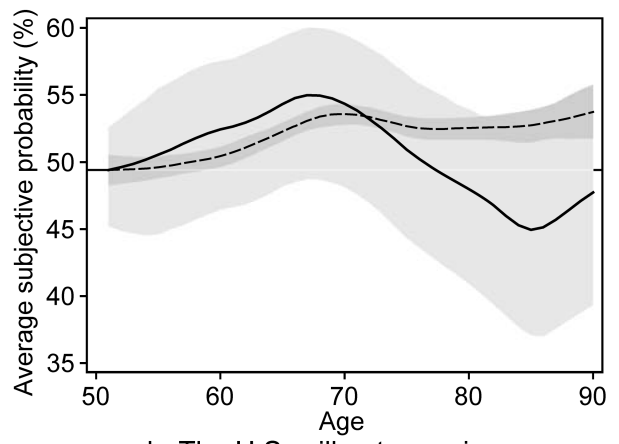

b. The U.S. will not experience economic depression

- - - cross-sectional profile - cumulative slopes

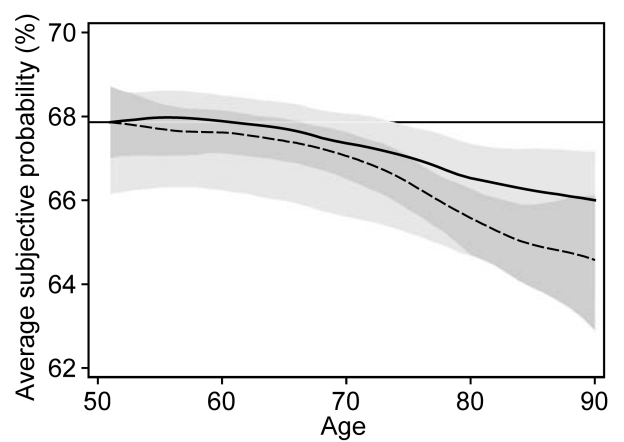

c. Sunny day tomorrow (movers excluded)

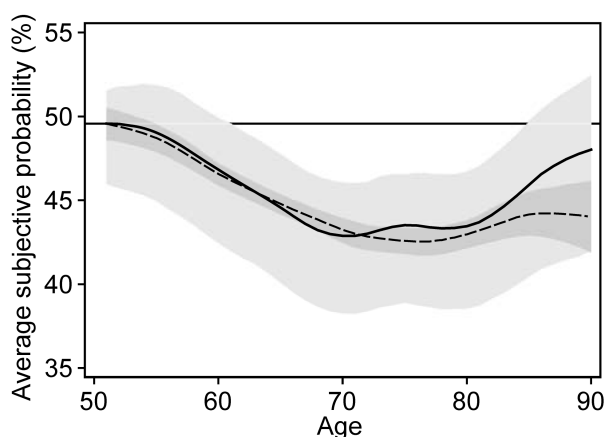

d. Income will keep up with inflation

- - - cross-sectional profile — cumulative slopes

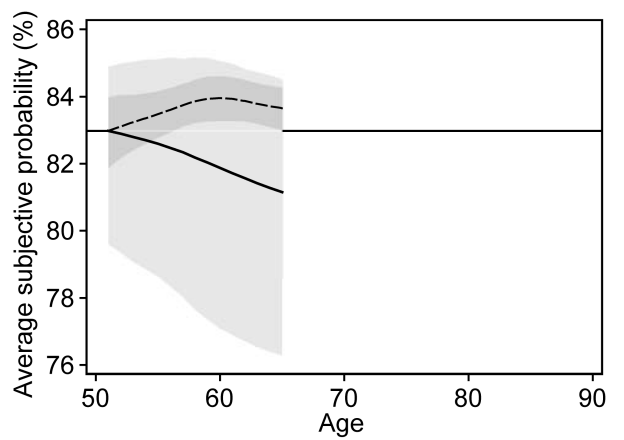

e. Will not lose job

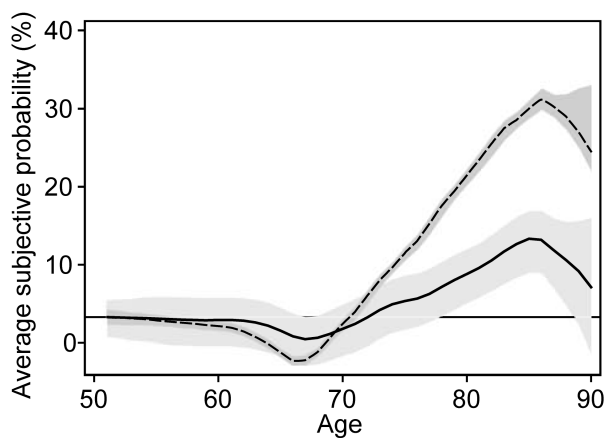

f. Survival to a specific age (difference from life table probabilities) - - - cross-sectional profile — cumulative slopes

Fig. 9.1 Age profiles of optimism 
age profiles are negative, the solid profiles are also significant and negative. Although it is not statistically significant, the divergence between the cross-sectional profiles of the cleaner age profiles of job loss expectations is consistent with selection, as this question is only asked of employed individuals, and individuals with higher expectations are more likely to stay employed than those with lower expectations.

In contrast to the other five variables, the cross-sectional profiles of survival expectations already take selection and cohort effects into account and thus represent the true age effects. Recall that we transformed the answers to the survival question by determining the difference between the respondent's answer and the probability implied by life expectancy tables. Those life table probabilities are already conditioned on cohort (as they are calculated separately for each year) and selection (as they show the probability of survival conditional on being alive for every year of age). In contrast, the profile built up from cumulative differences is biased in this case because it is conditional on survival to the next survey wave. In that way, for each year of age this method selects people with above average survival probabilities. Because these respondents had higher than average probabilities to begin with, the change in their probability is smaller. Because mortality and therefore selection accelerates with age, the divergence between the two lines increases with age.

The increase in optimism about life expectancy for those over age seventy shown by the dashed line in figure 9.1f. is consistent with the findings of Hudomiet and Willis (2013) for HRS and Hurd, Rohwedder, and Winter (2005) using SHARE data from Europe. This phenomenon is not driven by 50 percent answers. ${ }^{7}$ We can only speculate about the causes of this increase. This increase may be specific to survival: the true probability of survival declines rapidly, and an individual's expectations may not keep up with that decline. This pattern is also consistent with Carstensen's (2006) theory that the elderly increasingly focus on emotionally rewarding short-term goals. For example, their optimistic survival beliefs may allow them to focus on planning a trip or anticipating the birth of a grandchild without worrying about the possibility that they may not live to experience that pleasurable event. From an economic perspective, in the absence of full annuitization, it is rational for an individual to maintain a buffer of wealth as a precaution against outliving one's assets. Optimism about life expectancy could represent a short-hand way of dealing with uncertainty about the length of life by signaling a need to maintain more wealth than would be required based on the more realistic expectations contained in life tables.

7. While 50 percent is close to the "right" answer, based on life table to the survival question on average at younger ages, the right answer becomes substantially smaller at older ages. If people's propensity to say 50 percent increased with age as a result of increased uncertainty, that would show up as increased optimism. However, the increase in optimism remains strong when the 50 percent answers are discarded altogether, as found earlier by Hurd and Rohwed$\operatorname{der}(2006)$. 


\subsubsection{Age Profiles of Uncertainty}

We now present the age profiles of our measures of uncertainty. Figure 9.2 shows our preferred measure, the fraction of "don't know" and 50 percent answers, while figure 9.3 shows the fraction of "don't know" answers only. Similarly to the optimism measures, these are adjusted by the year-month of the interview according to formula (2). Also, similarly to the optimism measures, we discarded the sunny day answers of movers. However, in contrast to the optimism measures, we did not adjust survival expectations to life table probabilities here to retain the 50 percent answers.

The cross-sectional age profile of uncertainty is positive in four cases, zero for job loss expectations, and nonmonotonic for survival expectations. Whenever the cross-sectional profile of uncertainty is monotonically increasing, the cleaner age profile is also increasing. While the increase in the cleaner age profiles is statistically significant in only one of those four cases, it is jointly significant for the other three as well. The least precise estimated increase is for job loss expectations, which is only reported until age sixty-five.

When uncertainty is measured by the fraction of "don't know" answers only, uncertainty in survival expectations declines more strongly. Taken together, these results suggest that there is a general increase in people's propensity to give 50 percent and "don't know" answers with age, but the tendency to answer "don't know" as opposed to 50 percent increases significantly with age.

Survival expectations do not exhibit a positive effect: uncertainty does not change significantly until age seventy, and after that point it decreases. This pattern is largely driven by the 50 percent answers, which are responsible in part for the mirroring age profile of survival optimism. When uncertainty is measured by the fraction of "don't know" answers only, uncertainty in survival expectations increases significantly with age, similarly to the other expectations measured here.

Taken together, these results suggest that aging may have a generally negative effect on optimism and a generally positive effect on uncertainty, although these effects are not universal. In the remainder of the chapter we investigate the role of cognitive decline in explaining these general age effects.

\subsection{Cognitive Decline}

Cognitive functioning declines with age over the age range of our sample. Fluid aspects of intelligence - the ability to think and reason - peak in early adulthood and decline afterward, while more crystallized aspects - acquired knowledge - may continue to improve throughout much of old age and only begin to decline later (Horn and Cattell 1967; Horn and McArdle 2007; McArdle and Willis 2011). The decline in fluid cognitive functioning is a normal phenomenon over the age range of our sample, but some people 


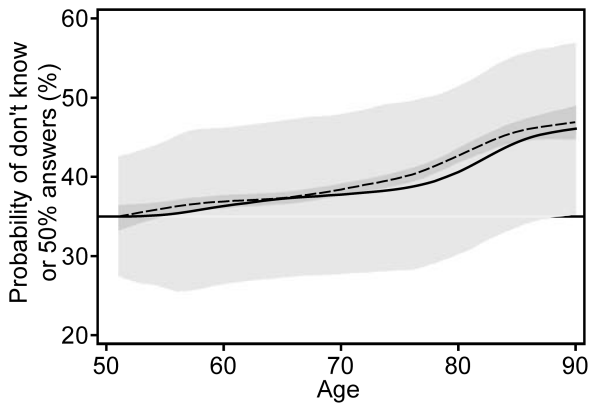

a. The stock market will go up

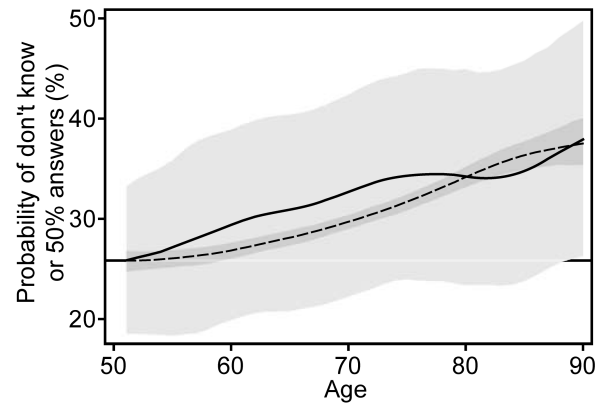

b. The U.S. will not experience economic depression - - - cross-sectional profile - cumulative slopes

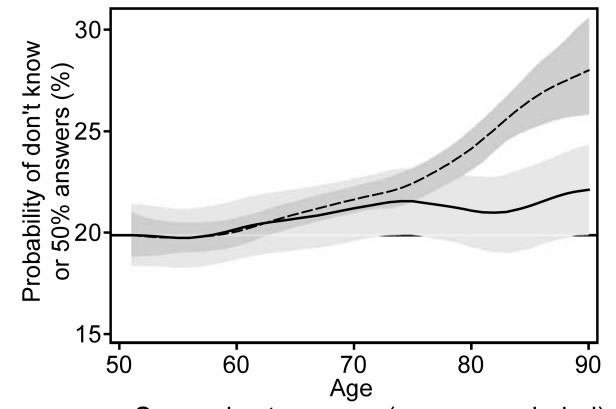

c. Sunny day tomorrow (movers excluded)

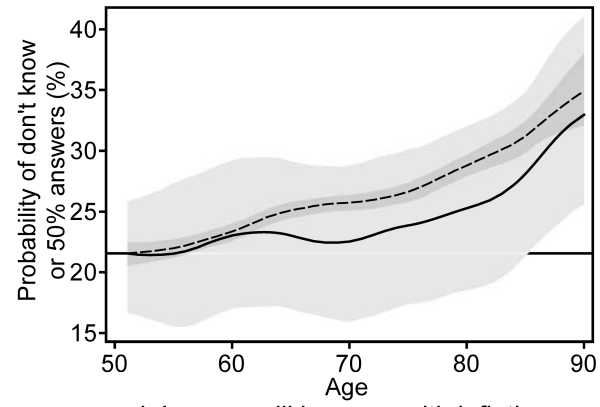

d. Income will keep up with inflation - - - cross-sectional profile - cumulative slopes

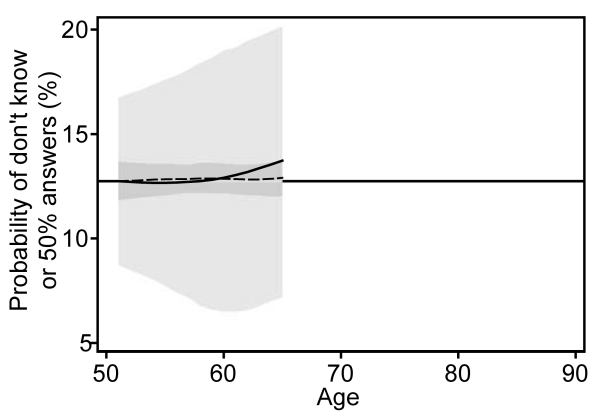

e. Will not lose job

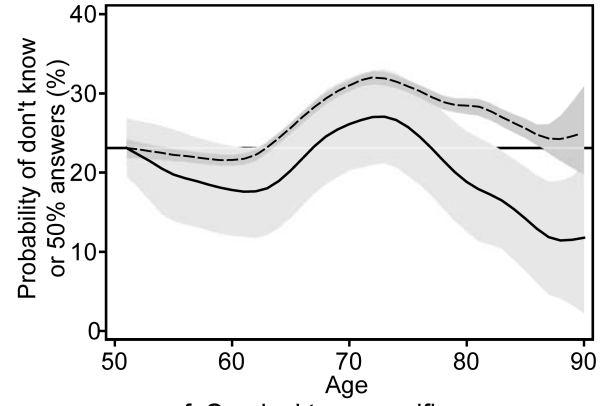

f. Survival to a specific age

- - - cross-sectional profile — cumulative slopes

Fig. 9.2 Age profiles of uncertainty 


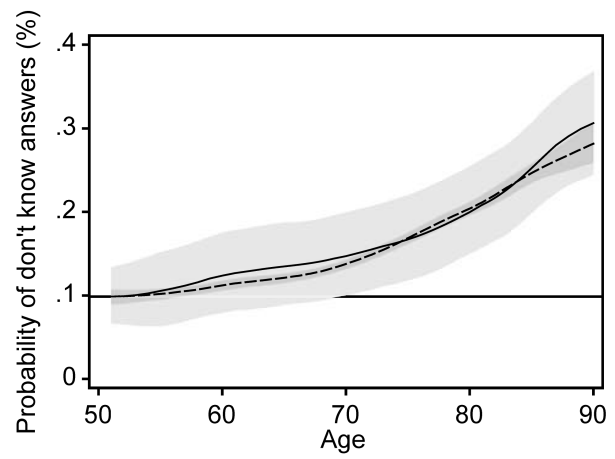

a. The stock market will go up

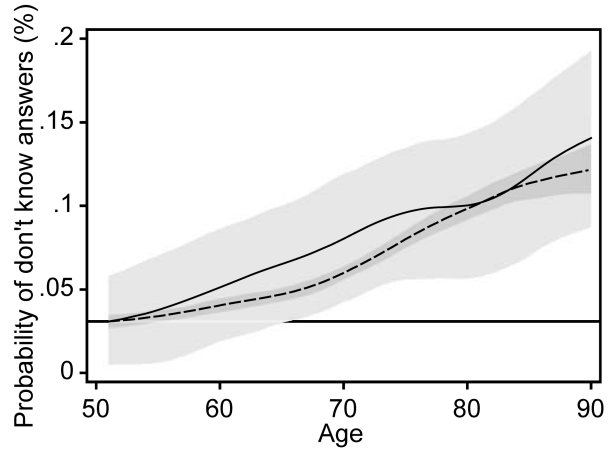

b. The U.S. will not experience economic depression

- - - cross-sectional profile - cumulative slopes

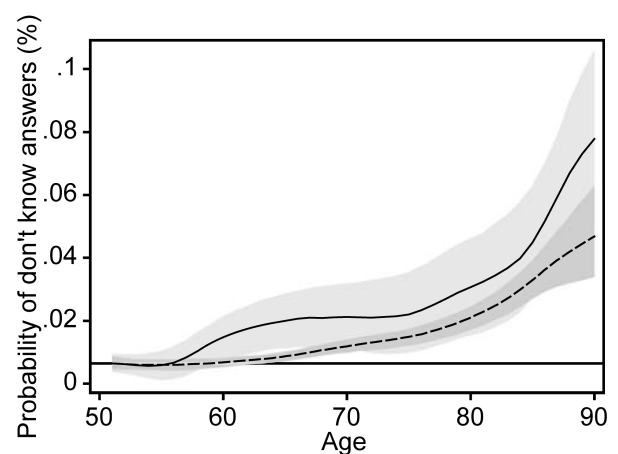

c. Sunny day tomorrow (movers excluded)

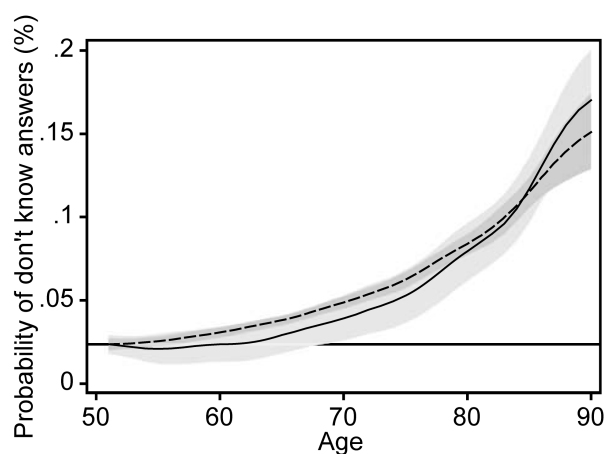

d. Income will keep up with inflation - - - cross-sectional profile — cumulative slopes

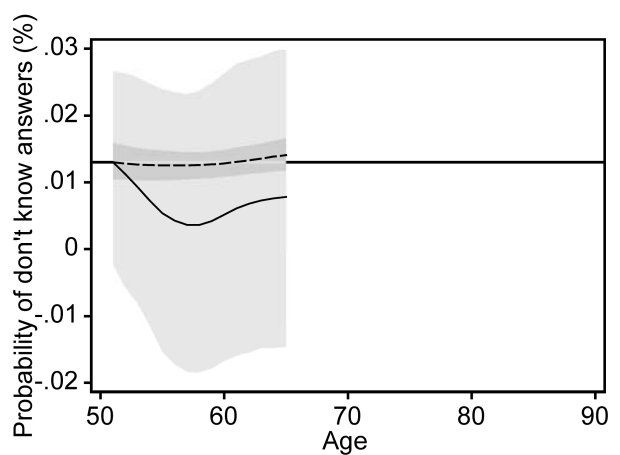

e. Will not lose job

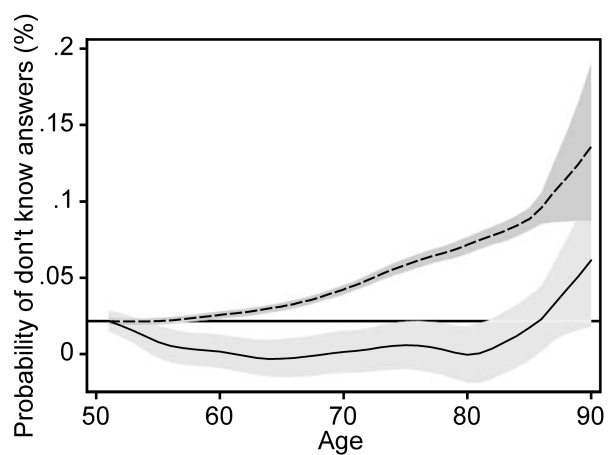

f. Survival to a specific age - - - cross-sectional profile - cumulative slopes

Fig. 9.3 Age profiles of the fraction of "don't know" answers 


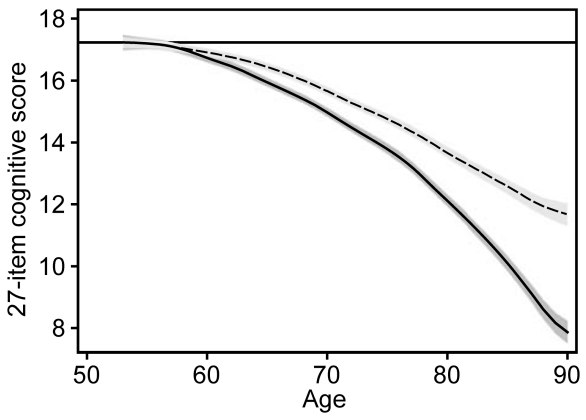

27-item cognitive score

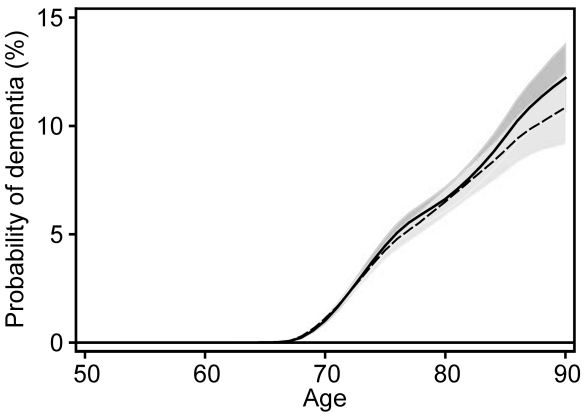

Predicted probability of dementia one year after interview (Hurd et al. 2013)

- - - cross-sectional profile — cumulative slopes

Fig. 9.4 Age profiles of cognitive decline

experience abnormally strong declines due to dementia. Most people do not experience dementia, but even among those who experience normal declines, the rate of cognitive decline can vary considerably.

Short-term memory and awareness follow age patterns that are very similar to fluid aspects of intelligence (McArdle et al. 2002.) Our twenty-seven-score measure of cognitive functioning is a combined measure of short-term memory, awareness, and numerical reasoning. Therefore, this measure should exhibit age patterns similar to those of fluid intelligence: apart from the onset of dementia, the measure should show a steady and relatively stable decline. Agarwal et al. (2009) show that three out of the four HRS tests that we use exhibit this age pattern.

Figure 9.4 shows the age profile of the twenty-seven-score measure of cognitive functioning and the probability of dementia. For each measure, we show both the cross-sectional profiles and the age profile built from cumulative slopes (as defined in equation [1]). The left panel is analogous to the graphs presented by Agarwal et al. (2009) in their figure 4, but we use slightly different samples, a combined measure, and also show confidence intervals. Despite these differences, the left panel of the figure presents a very similar picture to those presented by Agarwal et al. (2009). The cognitive score exhibits a steady decline with age, the cross-sectional profile is above the pure age profile, and the divergence between the two is greater after age seventy-five. These results suggest a strong and steady cognitive decline on average, as well as positive selection based on cognitive capacity that becomes stronger with age. These figures are also consistent with increasing fluid cognitive scores across birth cohorts, known in the psychology literature as the "Flynn effect" (Flynn 1987).

The right panel of figure 9.4 presents an analogous graph featuring the estimated probability of dementia. The age profile of the predicted proba- 
bility of dementia shows a steady and strong increase after age sixty-seven (the probability is zero earlier). Similarly to the cognitive score, the crosssectional profiles show a flatter profile indicating positive selection or positive cohort effects, but this difference is not statistically significant.

The age pattern of cognitive decline and the general age patterns of expectations exhibit some symmetries: cognitive functioning and optimism (in most domains) decline with age, while uncertainty appears to increase with age. These symmetries may suggest direct relationships. However, the theoretical arguments for these relationships are not conclusive. On the one hand, this relationship seems natural as forming expectations is a cognitive exercise. On the other hand, one important cognitive aspect of expectations is people's knowledge about the domain of the phenomena. Knowledge is a crystallized form of intelligence, and crystallized intelligence does not decrease together with fluid cognitive functioning (Horn and Cattell 1967). Theoretical arguments by cognitive psychologists and economists (McArdle and Willis 2011) as well as neuroscientists (Reuter-Lorenz and Park 2010) suggest that crystallized intelligence may remain high even if fluid intelligence experiences a steady decline and, in addition, may help compensate for this decline. Moreover, aging may also affect preferences (see, e.g., Carstensen 2006) that can influence the incentives to acquire and process information and knowledge that shape expectations. Therefore, the effect of aging on expectations may operate through mechanisms that are not directly related to the decline in cognitive functioning.

We investigate this question making use of individual heterogeneity in the rate of cognitive decline. If cognitive decline leads to changes in optimism and uncertainty, people who experience stronger declines in cognitive functioning should experience more pronounced changes in optimism and uncertainty.

\subsubsection{Measurement Issues and the Risk of Spurious Relationships}

Unfortunately, if heterogeneity in cognitive decline and changes in expectations are measured in the same survey, their measured relationship may be spurious. Heterogeneity in measured changes of cognitive functioning includes variations due to short-term idiosyncratic factors and to pure measurement errors, in addition to true variations in the rate of cognitive decline. For example, as we will demonstrate, the wave-to-wave change in the cognitive score is often positive due to short-term variations as opposed to genuine improvements in cognitive abilities. Similar idiosyncratic variations are likely to influence survey answers to expectation questions. Therefore, it may be problematic to perform a joint analysis of these variations. In this section we document the extent of the problem and propose a measurement strategy that minimizes the problem.

To facilitate this discussion, we label all additional variation as the "noise" and true variation in cognitive decline as the "signal." Noise may distort the 
measured relationship between cognitive decline and expectations measured by the same survey in two ways. First, if noise in cognitive decline is independent of potential measurement error in the optimism and uncertainty measures, a regression with cognitive decline on the right-hand side will produce slope coefficients that are biased toward zero. This is a classical measurement error situation. However, noise in cognitive decline and noise in the optimism and uncertainty measures may be correlated. Variations in the effort required to answer survey questions from interview to interview for the same individual might lead to such a correlation. An interview with a lower input of effort by the respondent may result in lower scores for the cognitive tests and a higher propensity to answer "don't know" or "50 percent" to the expectation questions. This may lead to a spurious relationship between measured cognitive decline and measured uncertainty. Whether the noise is classical or correlated, the magnitude of the bias is larger if the noise-to-signal ratio is larger.

Note that these arguments may be relevant for the relationships among changes in other variables measured in the same survey if they are also subject to considerable noise. The issue is not whether the relationships are causal but whether the relationships measured by survey data correspond to relationships between the phenomena themselves as opposed to pure survey noise. There is no foolproof way to address survey noise. Our strategy in this chapter is to construct measures of age-related changes that are least affected by survey noise and to search for circumstantial evidence indicating whether the measured relationships could be driven by noise.

A natural analysis would relate wave-to-wave changes in measured expectations to wave-to-wave changes in cognitive scores. However, those firstdifferenced measures are also the most affected by survey noise. To mitigate the bias, we carried out our analysis on individual slopes. For each individual, we regressed the cognitive score on the individual's age at the time of the interview (measured to monthly precision) and saved the coefficients from that regression. For each individual, the slope of cognitive decline is the slope coefficient from this regression. Then, we performed similar individual regressions for each expectation measure after adjustments to the year-month of the interview and the other adjustments described earlier. ${ }^{8}$ We restricted the individual regressions to individuals with three or more observations. The maximum number of observations is seven for the cognitive score and smaller for the measures that are not available in every survey wave.

Regressions of the slopes of expectations on the slopes of cognitive decline identify the relationship based only on between-individual heterogeneity. The slopes of cognitive decline are characterized by a lower noise-to-signal

8. These include flipping of negative events, defining survival optimism as the difference from life tables, and restricting sunshine data to those who do not change residence. 
Table 9.3

Summary statistics of the age-adjusted first difference in cognitive score and the age-adjusted slope of cognitive score

\begin{tabular}{lcc}
\hline & First difference $^{\mathrm{a}}$ & Slope measure $^{\mathrm{b}}$ \\
& $(1)$ & $(2)$ \\
\hline Mean & -0.22 & -0.22 \\
Standard deviation & 1.85 & 0.52 \\
\hline
\end{tabular}

${ }^{\text {a }}$ Wave-to-wave change in the cognitive score divided by wave-to-wave change in the age of the respondent.

${ }^{b}$ Estimated individual slopes of the cognitive score from individual-specific regressions on age at the time of the interview.

ratio than the wave-to-wave changes in the cognitive score (see the following evidence). Therefore, regressions on the slope measures produce estimates that are less biased than the results of regressions on the first differences. The bias is reduced further if the sample is restricted to individuals with a relatively large number of observations used in the individual regressions that estimate the slope measures. Individual slopes are analyzed in the spirit of the latent growth modeling technique used by McArdle et al. (2002).

Table 9.3 presents summary statistics of the age-adjusted first difference of the cognitive score variable (the wave-to-wave difference of the cognitive score divided by wave-to-wave difference in age) and the age-adjusted slope measure of the cognitive score (the slope coefficients of the individual regressions of cognitive score on age). The mean of the cognitive change measures remains the same. Aging one year is associated with an approximately 0.2 percent decline in the cognitive score. At the same time, the variance of the first difference measure is substantially higher than the variance of the slope measure.

Figure 9.5 shows histograms of the first-differenced measure of cognitive decline and the individual slope measures. The graph of the slope measures includes the histogram of all slope estimates as well as the histogram of the slope estimates from the subsample of individuals with the maximum number of observations, which is seven. Within each histogram, lighter colors indicate positive measured changes in cognitive functioning. Positive changes are unlikely to reflect true long-term changes in cognitive functioning because the cognitive measure assesses fluid aspects of cognitive functioning, which typically do not improve with age in this age range.

The histograms show the wide dispersion of the first-differenced measure and the narrower dispersion of the slope measure. The distribution of the slope measure is even more concentrated if it is restricted to the subsample of respondents with the maximum number of observations. There is some excess mass around zero for the first-differenced measure, which is an artifact of normalizing the change in the cognitive measure. As this measure is a small integer, noninteger changes in age do not change the cognitive 

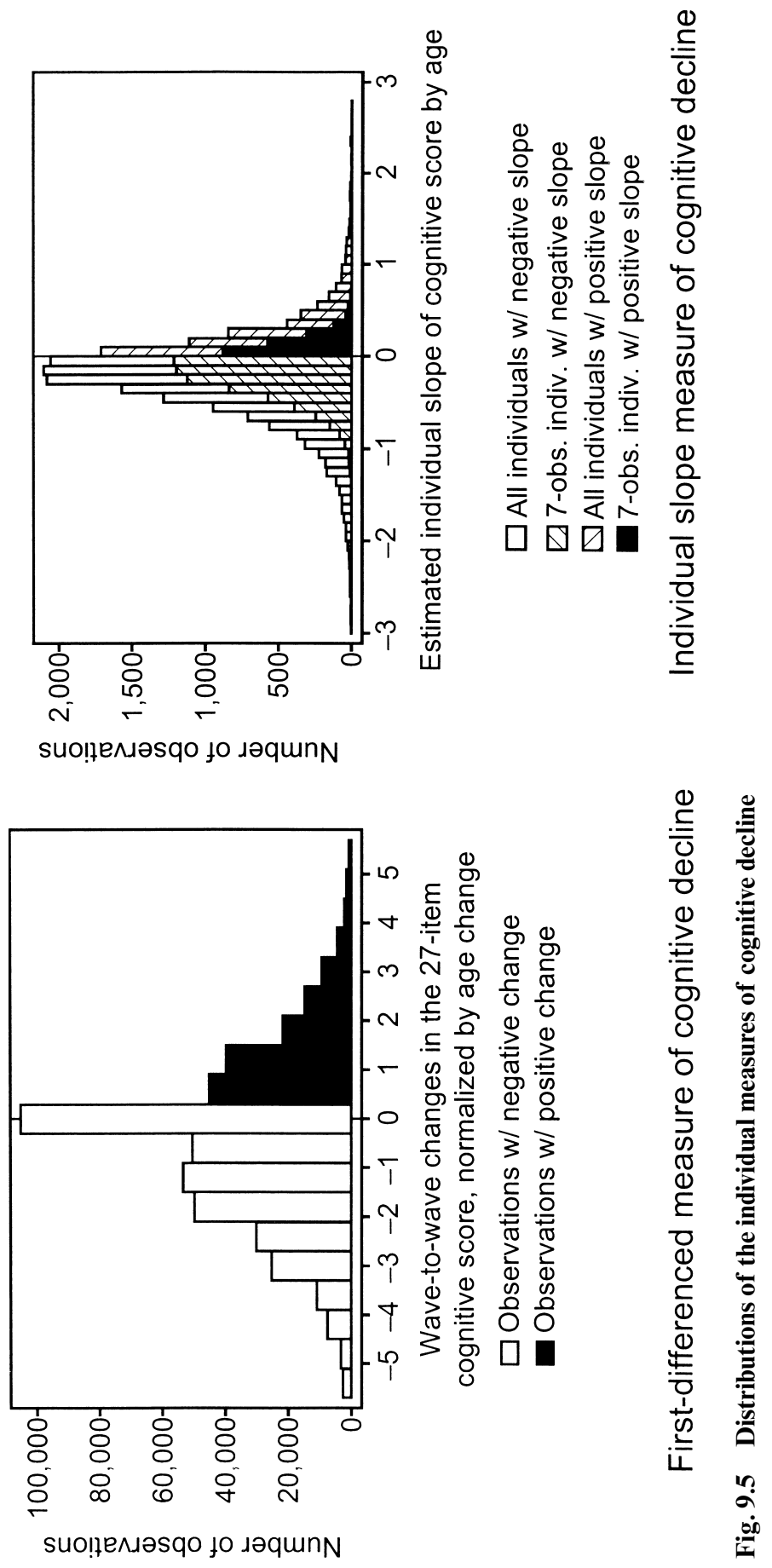
score but lead to a spread of all nonzero changes. In addition to the wide distribution, the histograms highlight the nonnegligible fraction of positive measured changes. The fraction of positive changes is 39 percent for the first-differenced measure, 29 percent for the slope measure, and 25 percent for the slope measure in the maximum-observation subsample. The histograms support the assumption that the slope measures have considerably lower noise-to-signal ratios, especially when restricted to the maximumobservations subsample.

We can examine changes in the method of data collection to provide further evidence of the noise in the cognitive measures. We analyze the effects of wave-to-wave changes in the interview mode and in the identity of the interviewer at the level of the variation in cognitive measures. In the HRS, the baseline interview with each respondent is a personal interview; these occurred in 1992, 1998, 2004, and 2010. Before 2006, subsequent interviews were normally conducted by telephone for respondents under age eighty and in person for those over age eighty. However, a small number of respondents request a change from the normal mode and HRS honors these requests. In 2006, the HRS initiated an "enhanced face-to-face" interview to collect biomarkers and physical performance data. A random half of the longitudinal sample was selected for the enhanced personal interview in 2006 with the other half receiving a personal interview in 2008. The half selected for enhanced interviews in 2006 received second enhanced interviews in 2010. The interview mode changes between two interviews almost half of the time, and these changes are roughly equally split between changes from telephone to personal and vice versa. In 70 percent of cases, the interviewer also changes from wave to wave. These two changes are weakly correlated: a change in the interviewer is 12 percentage points more likely when the interview mode changes.

Changes in the survey mode and in the interviewer may increase noise in measures for a variety of reasons. Both the interview mode and the match between interviewers and respondents can affect the noise in survey answers. Effects on the effort that respondents exert in answering the survey questions may lead to variations in the cognitive score and in the propensity to provide uncertain answers to the expectation questions. Effects on the attitudes of the respondents may lead to noise in the optimism of the expectation answers.

Table 9.4 presents the results. The first column shows the results of regressions on the first difference of the cognitive score. The second column shows results of regressions on the squared residuals of the regression in the first column. Each regression has four main right-hand-side variables capturing whether there was a change in the interview mode, whether the interview mode was personal and unchanged, whether the mode of interview changed from personal to telephone, and whether it changed from telephone to personal. The regressions control for a full set of year-of-age dummies and 
Changes of interviewers, changes of survey modes, and the mean and variance of changes in the cognitive score

\begin{tabular}{lcc}
\hline & $\begin{array}{c}\text { First-differenced } \\
\text { cognitive score }\end{array}$ & $\begin{array}{c}\text { Squared residual from the } \\
\text { regression on the first- } \\
\text { differenced cognitive score }\end{array}$ \\
\hline Change of interviewer & -0.03 & $0.3^{* * *}$ \\
Interview mode unchanged & $(0.01)^{*}$ & $(0.1)$ \\
Personal & 0.03 & $-0.7^{* * *}$ \\
Interview mode change & $(0.02)$ & $(0.1)$ \\
Personal to phone & 0.12 & $0.3^{* * *}$ \\
Interview mode change & $(0.02)^{* * *}$ & $(0.1)$ \\
$\quad$ Phone to personal & -0.02 & 0.1 \\
Year of age fixed effects & $(0.02)$ & $(0.1)$ \\
Year-month fixed effects & YES & YES \\
$R$-squared & YES & YES \\
Number of observations & 0.006 & 0.014 \\
\hline
\end{tabular}

Note: Standard errors clustered at the household level in parentheses.

***Significant at the 1 percent level.

**Significant at the 5 percent level.

*Significant at the 10 percent level.

year-month dummies to capture age effects and time effects that may be correlated with changes in the interviewer and the survey mode.

The most important results of table 9.4 are in column (2): wave-to-wave changes in the interviewer and some changes in survey mode are associated with a significant increase in the variance of the measured change of the cognitive score. Compared to individuals with phone interviews with the same interviewer in both waves, the variance of the change in the cognitive score is lower for individuals with personal interviews in both waves, and it is higher for individuals whose interview mode changes from personal to telephone. A change in the interviewer is also associated with a greater variance. Column (1) shows that the decline in the cognitive score is less negative for individuals whose interviews change from personal to telephone and that a change in the interviewer is weakly associated with a stronger decline of 0.03 . While some of the associations with the first-differenced score may indicate causality from cognitive decline to changes in data collection, the association of these changes with a higher variance is consistent with some of the variance resulting from survey noise.

We have estimated similar regressions for changes in optimism and uncertainty. The results of those regressions indicate that the associations with changes in survey mode are mixed, but that a significant association exists between the change in the interviewer and the variance of the first-differenced measures of optimism and uncertainty for many expec- 
tation questions. Together with the results of table 9.4, these indicate the association between expectation measures and cognitive decline measured by regressions estimated by first differences may be identified in part based on variations in noise. As noise in first-differenced cognitive scores is correlated with noise in first-differenced dependent variables, coefficients in such regressions are likely to be biased away from zero. The results of those regressions indicate strong negative associations between the change in the cognitive score and changes in optimism and positive associations between the change in the cognitive score and changes in uncertainty. However, the potentially spurious nature of those associations is supported by the fact that the estimated relationships are very similar if they are identified solely from positive changes in the cognitive score, where the variation is likely to be dominated by noise.

\subsubsection{Expectations and Cognitive Decline}

Our preferred specification for estimating the association of changes in expectations with cognitive decline uses individual slope estimates instead of first differences. Because of their lower noise-to-signal ratio, using the individual slope estimates in regressions of expectations on cognitive score are likely to result in a lower bias. Tables 9.5 and 9.6 show the results of the regressions in which the left-hand-side variables are the individual slope estimates of optimism and uncertainty, respectively, for the six probability questions analyzed here.

In each regression, the main right-hand-side variable is the slope of the cognitive score, which we multiplied by negative one to represent cognitive decline. A positive coefficient would imply that cognitive decline is associated with an increase in our measures of optimism or uncertainty. This coefficient is identified from variations in the average rate of cognitive decline across individuals. That rate of decline is estimated from separate regressions for each individual with three to seven observations. The bias of the coefficient on this variable should be smaller for individuals with seven observations than for individuals with fewer observations, a fact that we will take advantage of when conducting robustness checks.

The individual slopes of left-hand-side variables and the cognitive score are calculated from individual regressions with age on the right-hand side, measured to monthly precision. We adjusted each optimism and uncertainty measure to deviations from year-month fixed effects before estimating the individual slopes. The rest of the right-hand-side variables consist of the age of the individual at baseline (the first observation of the cognitive score) normalized to zero at age fifty-one; the dependent variable at baseline as predicted from the individual slope regressions (normalized to have a mean of zero); and the cognitive score at baseline as predicted from the individual slope regressions (normalized to have a mean of zero).

The constants of the regressions show the changes in the left-hand-side 


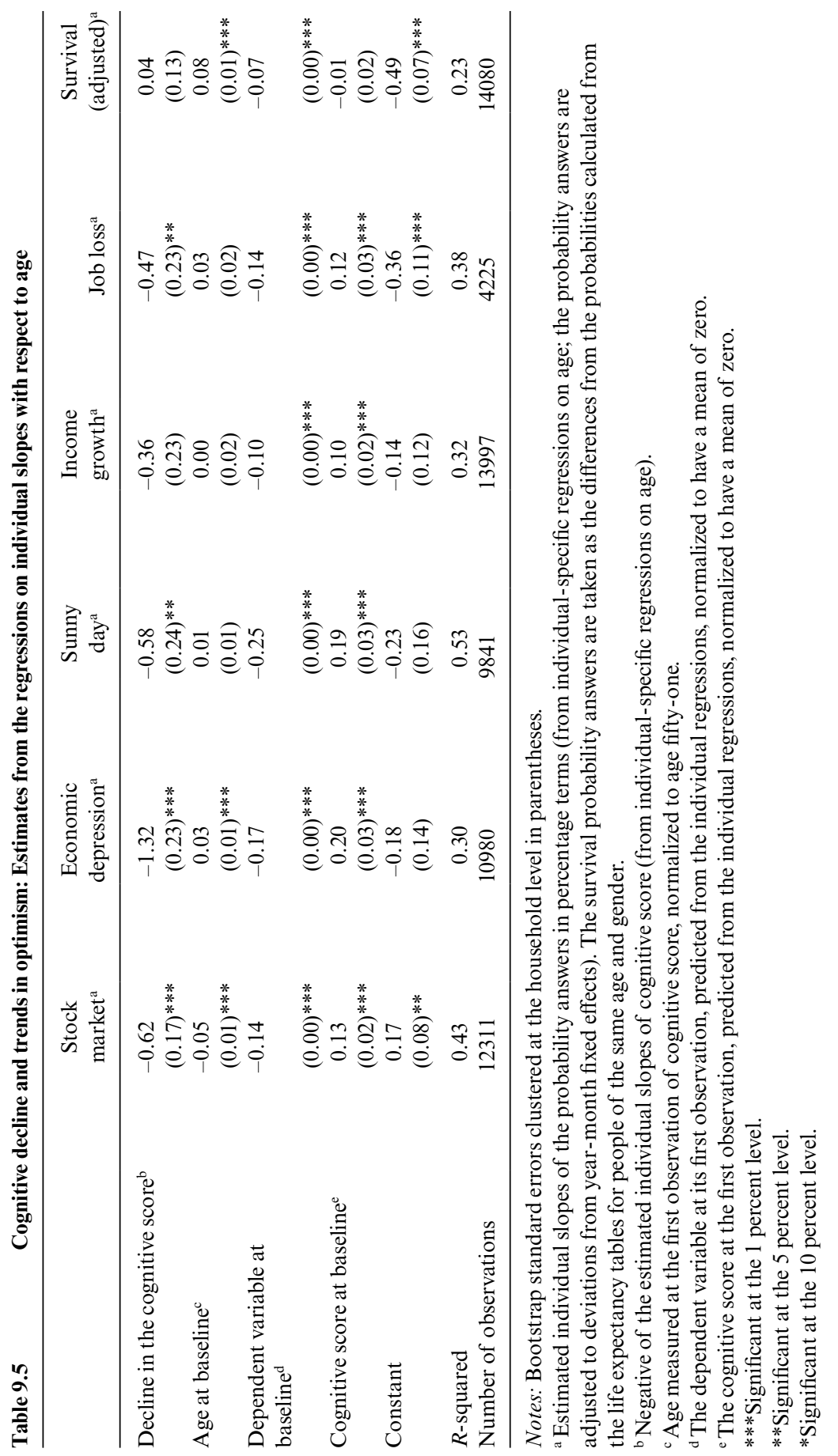


variables that correspond to aging by one year, measured at age fifty-one, if the dependent variable and the cognitive score are at their average baseline values and if cognitive decline has a slope of zero. Associations with age beyond age fifty-one are allowed to be nonlinear. The coefficient of the age at baseline variable corresponds to that nonlinearity, indicating how the age-related change in the left-hand-side variables changes with age. The coefficient of the dependent variable at baseline shows the relationship between the individual slopes of the left-hand-side variables and their initial values (holding the other variables constant), where the initial value is predicted from the individual-specific regressions. Mean reversion, whether due to noise or to other idiosyncratic variations in the left-hand-side variables, would imply a negative coefficient. The coefficient of the cognitive score at baseline variable shows the correlation between the average change in the left-hand-side variable and the cognitive score at the first observation (holding the slope of the cognitive score and the other variables constant), where the value at the first observation is again predicted from the individual regressions. Here, mean reversion is captured by whether this coefficient has the same sign as the coefficient of the cognitive decline variable because the decline variable is the negative of the average change of the cognitive score.

The results show a modest but often statistically significant association between the rate of cognitive decline and the average change in expectations for all probability questions except for survival. To understand the magnitudes of the coefficients, note that the average rate of decline in the cognitive score for every additional year of age is 0.2 (this is also the 55th percentile of the distribution). Individuals with a 0.1 point higher rate would be at the 70th percentile of the distribution of measured cognitive decline. The coefficients of the cognitive decline variable suggest that individuals with a 0.1 point higher rate of cognitive decline experience, on average, a 0.04 to 0.13 percentage point decline in their answers for every additional year of age. This accounts for 1 to 2 percent of the standard deviation in the slope measures of the left-hand-side variables. Calculated for the twelve-year horizon that the data span, individuals with a cognitive decline at the 70 th percentile would experience a 0.5 to 1.5 percentage point decline in their probability answers, which is a small but nonnegligible change. Note that the magnitudes are difficult to appreciate because these coefficients may still be biased in unknown directions. The robust negative coefficients of the dependent variable at baseline suggest strong mean reversion, highlighting the importance of noise in the left-hand-side variables.

The exception to the negative association with optimism is the survival expectation: people with higher rates of cognitive decline do not experience any difference in the changes in their survival expectations from individuals with lower rates of cognitive decline. 
The coefficient of the baseline level of cognitive decline is positive in five of the six cases and zero for survival expectations. When positive, these partial correlations show that people with higher levels of cognitive functioning have lower rates of decline in optimism, holding the rate of decline and age constant. The coefficient estimates are positive whenever the coefficient of the cognitive decline is negative, providing additional support for the positive relationship between cognitive functioning and optimism. Note that our finding of mean reversion due to noise in the cognitive decline variable would result in the same sign of the coefficients of level and decline variables because the decline variable is the negative of the change. The fact that we do not find the same sign suggests that the noise in cognitive decline is not strong enough to produce such a mean reversion. Taken together, the coefficients of the cognitive decline variable and the cognitive score at baseline suggest a robust statistically significant relationship between cognitive decline and decreased optimism in five out of the six expectations we analyze.

The constants and coefficients of age in the optimism regressions provide a heterogeneous picture. Optimism about income growth and sunshine do not show significant dependence on age when cognitive decline and baseline cognitive functioning are held constant. Optimism about the stock market shows a weak positive relationship with age starting at age fifty-one that very quickly becomes negative, reaching a strong negative slope of negative one percent by age seventy, even when the rate of cognitive decline and the baseline level of cognitive functioning are held constant (at least as they are measured in our data). Optimism about job loss shows a negative relationship with age at age fifty-one that may or may not lessen with age, as the coefficient of age is positive but insignificant. The age profile of optimism about economic depression is flat at age fifty-one when the level and the change in cognitive functioning are held constant, but it exhibits a small but statistically significant increase at later ages, reaching a slope of 0.7 at age eighty. The estimated age profile of survival expectations is not affected by either the decline or the level of cognitive functioning, and thus it presents the same picture as figure 9.1 : survival optimism decreases initially but then increases at around age sixty-five.

Taken at face value, the estimates suggest that cognitive decline is associated with declining optimism in domains of private economic conditions, aggregate economic conditions, and sunny weather. While deteriorating private economic conditions may be affected by declining cognitive functioning for fundamental reasons, declining optimism in the more general domains is more likely to reflect some more general association between ageinduced decline in fluid cognitive functioning and less optimistic thinking in general. An increase in optimism about survival expectations with age is an important exception to this phenomenon. As discussed earlier, one reason for this may be based in the psychological tendency for older people to focus on emotional sources of future satisfaction without worrying about whether 
they will be alive to enjoy them. Another reason is that optimism about the chance of survival may serve as a heuristic device to help people maintain sufficient wealth to enable them to maintain their living standards should they survive to an exceptionally old age.

Some of the variation in observed cognitive decline is because some people suffer from dementia. Recall that we do not observe the expectations of the demented respondents, as they are not asked the probability questions. Instead, we use the predicted individual probability of the onset of dementia one year after the interview as estimated by Hurd et al. (2013). Unfortunately, a joint analysis of the decline in the cognitive score and the potential increase in the probability of dementia is not possible due to the strong correlation between these two characteristics and to the fact that a large portion of the variation in dementia probability is concentrated in the relatively small sample of individuals above seventy-five years of age. To determine whether the estimates in table 9.5 reflect associations with the onset of dementia, we reestimated all regressions on the subsample of respondents whose estimated probability of dementia remained below 5 percent in all survey waves. These results are very similar to those presented in table 9.5, with the exception of sunny day optimism, where the association with cognitive decline is not significant.

We performed several robustness checks. First, we restricted the sample to individuals with the maximum number of observations used in the slope regressions, which is seven for the cognitive score. As suggested by the right panel of figure 9.4, the noise in the slope estimates is substantially smaller in this subsample. The coefficients of the cognitive decline variable are very similar to, and in most cases stronger than, those presented in table 9.5. This suggests that the coefficients in table 9.5 represent genuine associations.

Second, we controlled for symptoms of clinical depression in both the first and last observations of each individual. This robustness check was motivated by the fact that cognitive decline is associated with deteriorating health conditions and that deteriorating health conditions may be responsible for the observed decline in the levels of expectations. That worry should be strongest for the survival expectations, and we do not see any association with cognitive decline despite this concern. However, some subtle changes may operate through depressive symptoms for all other expectations. Controlling for depressive symptoms should lead to weaker coefficients if that concern is warranted, but no such decrease is observed. Whether the estimate is performed for the whole sample, the sample with the maximum number of observations or individuals with low probabilities of dementia across all interviews, controlling for depressive symptoms does not change the results.

Finally, we reestimated all regressions on separate subsamples with declin- 
ing and increasing slope estimates for the cognitive score. As we have argued, positive slopes are more likely to reflect idiosyncratic positive changes in the measured cognitive score variable than genuine long-run improvements in cognitive functioning. Therefore, if the association between cognitive decline and declining optimism is genuine, it should be strong in the subsample of declining cognitive scores and weak in the subsample of increasing cognitive scores. These results are in line with these general expectations. In most cases, the coefficient estimates of the cognitive decline variable are stronger in the subsample of declining slopes than in the entire sample, and none of these estimates are statistically significant for the subsample with increasing slopes. The results of these robustness checks provide strong support for the relationship between cognitive decline and age-related decline in optimism for five out of the six expectation questions analyzed here. Expectations about survival remain an important exception.

After establishing some fairly general associations between cognitive decline and age-related changes in optimism, we turn to age-related changes in uncertainty. Table 9.6 presents results with a similar structure to table 9.5.

In contrast with the statistically significant results for optimism, the estimated association of the rate of cognitive decline with the rate of increase in uncertainty is not significantly different from zero in four out of the six cases examined here. In the two significant cases the sign is opposite: higher rates of cognitive decline seem to be associated with lower increases in the propensity to provide uncertain answers to the stock market question but with higher increases in the propensity to provide uncertain answers to the sunny day question. In cases where the coefficient of the cognitive decline variable is not significant, the coefficient of the baseline level of cognitive functioning is not significant either. In the other two cases, the coefficients of the levels strengthen the coefficients of the decline: a higher initial level of cognitive functioning is associated with a stronger increase in uncertainty about the stock market but a weaker increase in uncertainty about sunshine. These results suggest that there is no general tendency for age-related changes in uncertainty to be associated with cognitive decline, although cognitive decline may be associated with uncertainty with respect to specific events in specific ways.

The lack of a general association between cognitive decline and increasing uncertainty is confirmed by our robustness checks. The results are similar or even weaker when restricted to individuals with seven observations for the cognitive score or with low probabilities of dementia, and when the results are controlled for depressive symptoms. As an additional robustness check, we reestimated all regressions by measuring uncertainty as the propensity to answer "don't know" (ignoring the 50 percent answers). These results are even weaker, with no association observed between cognitive decline and uncertainty, even in the case of sunshine expectations. 


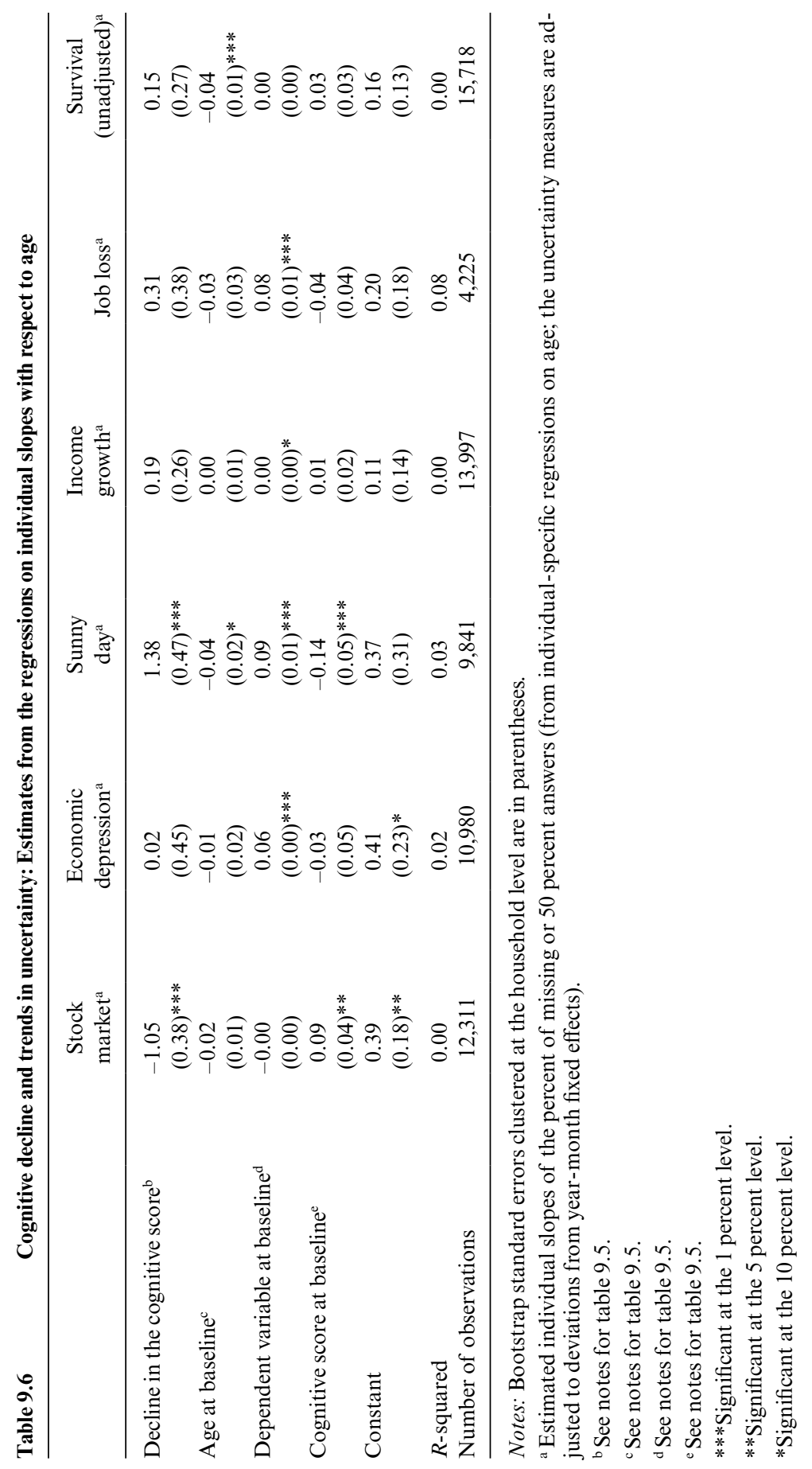




\subsection{Conclusion}

This is an exploratory study of the relationship between expectations, aging, and cognitive decline. We used data from seven waves of the Health and Retirement Study (HRS) to establish age patterns in optimism and uncertainty of expectations in six different domains: stock market returns one year in the future, the chance of a future economic depression, whether tomorrow will be a sunny day, whether one's income will keep up with inflation, job loss, and survival to a specific age. Respondents were asked to state their expected probabilities for the events in question. We measure optimism as higher subjective probabilities of positive events and uncertainty as a higher propensity to answer "don't know" or "50 percent."

We find that optimism decreases and uncertainty increases with age in three of the six domains, controlling for time, cohort, and selection effects. We also find that cognitive decline plays a modest but statistically significant role in explaining the decline of optimism in most domains. The important exception to both the effect of age and the role of cognitive decline is survival expectations: optimism about survival increases significantly with age, uncertainty appears to decrease, and cognitive decline plays no role in those effects. Somewhat surprisingly, cognitive decline does not seem to play a role in accounting for the increase in uncertainty in any of the domains that we investigate. We argue that the joint analyses of cognitive decline and changes in expectations that use person-specific slopes provide less scope for finding spurious relationships that would be more problematic in alternative models, and we provide several robustness checks to substantiate our findings on the association between cognitive decline and declining optimism.

Our finding of a general pattern of decreasing optimism and increasing uncertainty about sunshine, growth in real income, job loss, gains in the stock market, and economic depression is consistent with a pattern of cognitive decline that makes it more difficult for people to acquire and process knowledge and information about events in the world. To the extent that these patterns in the survey responses reflect beliefs that people act on in making decisions, we would expect to find that people act with greater caution and take fewer risks as they grow older. Agarwal et al. (2009) argue that declining cognitive capacity causes older people to make more mistakes in decision making. Our results on expectations suggest that older people may reduce the damage from mistaken decisions by attempting to avoid them altogether. For example, avoiding the purchase of a financial product that one does not understand may reduce the risk of being victimized by a scam, but that reduced risk must be balanced against the potential benefits that could be obtained if it is a good product. Increased pessimism and uncertainty would tilt this calculation in favor of avoiding the purchase.

Our finding of increased optimism about survival as people age may be an exception to the aforementioned analysis due to people's inability to adjust 
their expectations to the acceleration in risk of mortality at later ages. In a somewhat more speculative vain, our results can be interpreted as consistent with Carstensen's (2006) socioemotional selectivity theory of aging that posits that people become increasingly selective, investing greater resources in emotionally meaningful goals and activities because of an ever shorter time horizon before death. We speculate that optimism about survival allows the elderly individual to focus on emotionally rewarding short-term goals such as planning a trip or anticipating the birth of a grandchild without worrying about the possibility that they may not live to experience the pleasurable event. Optimism about survival may also serve an economic purpose as a heuristic that helps people to maintain a buffer of wealth as a precaution against outliving one's assets by giving greater subjective weight to the chance of an unusually long life.

It is important to stress that the findings in this chapter are exploratory and that our interpretation of those findings is speculative. We do believe that greater understanding of the ways in which probability beliefs are influenced by aging and cognition is a promising line of research. One priority for future research will be to link changes in beliefs to behaviors and decisions. 


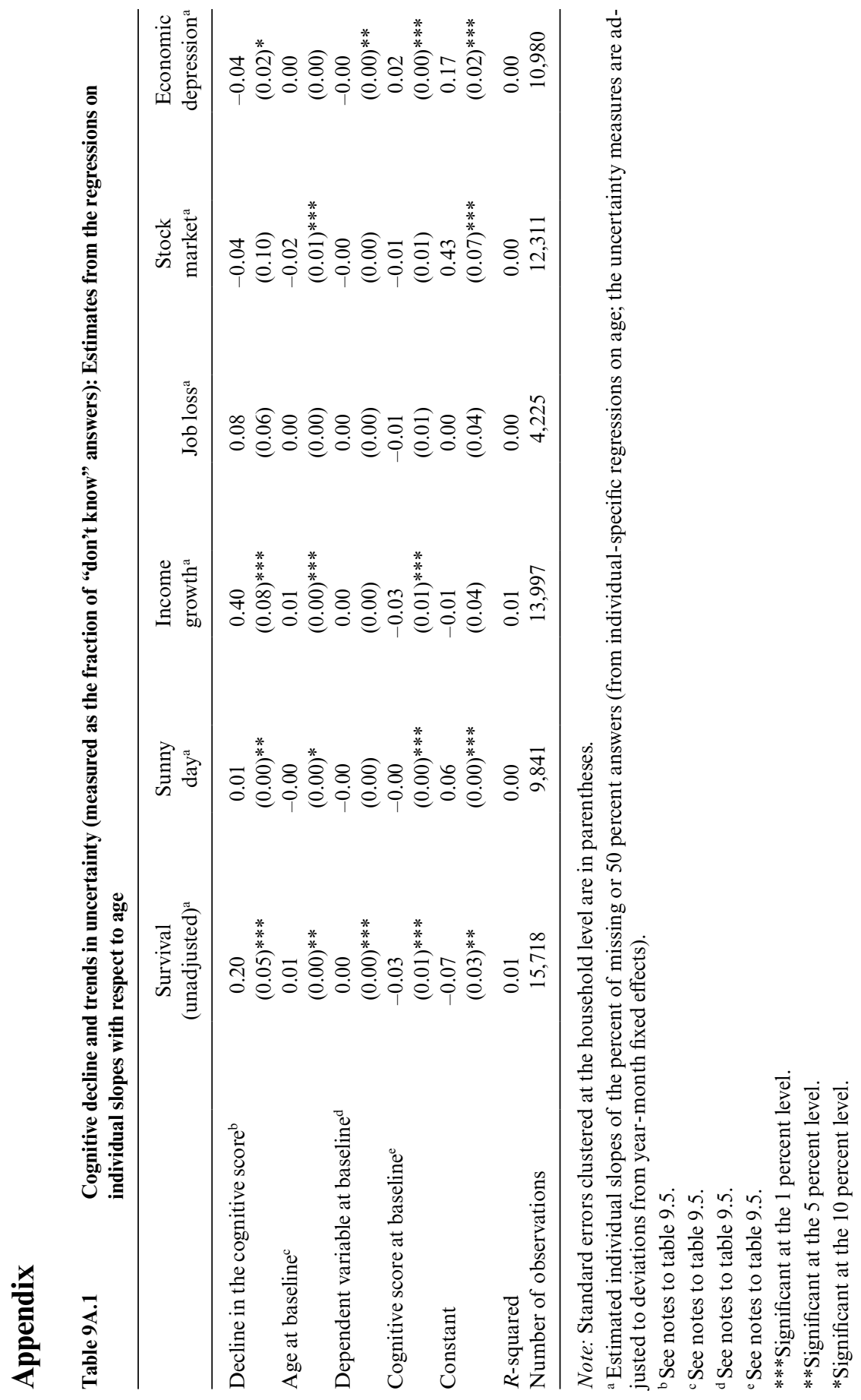




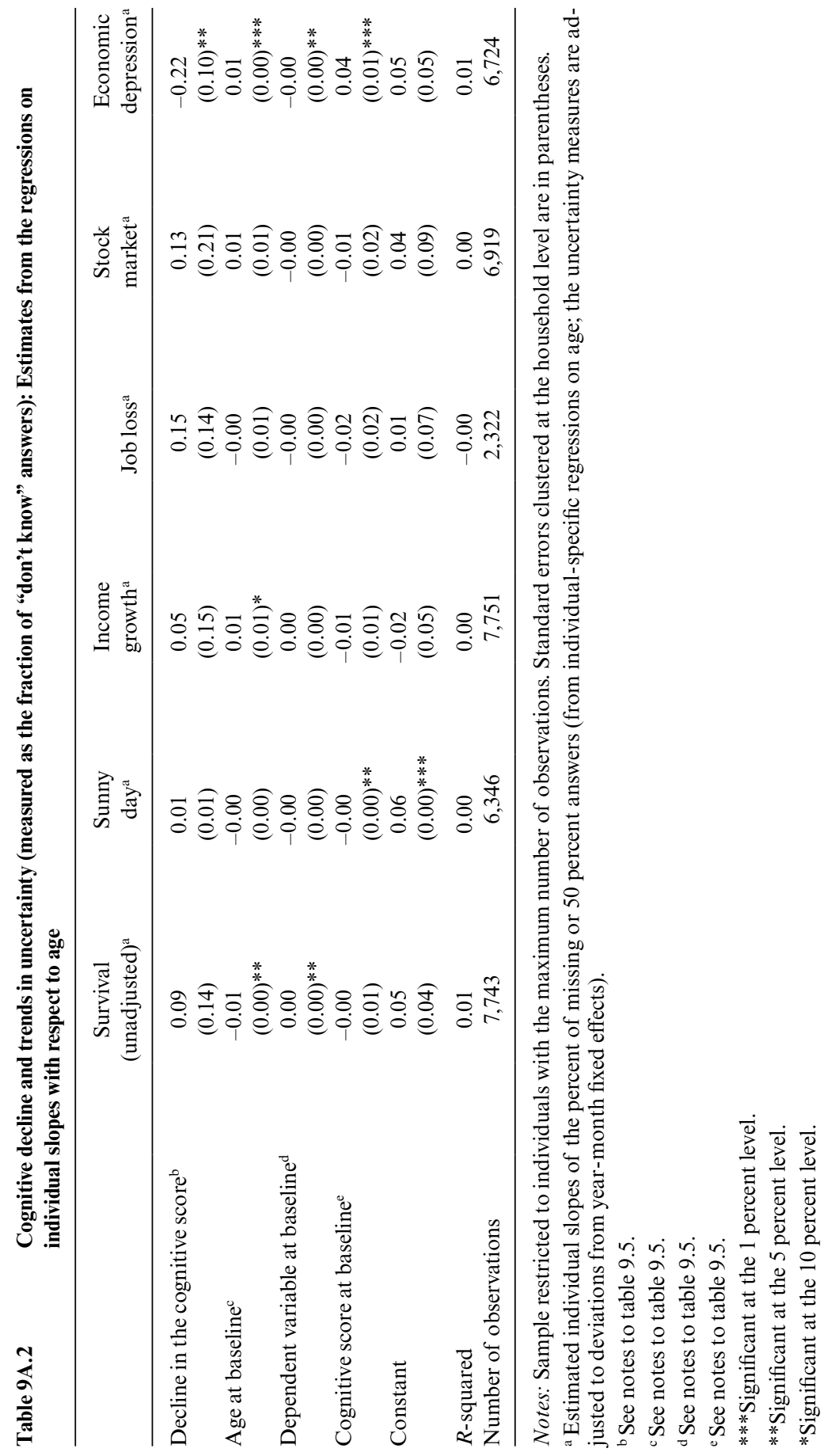




\section{References}

Agarwal, Sumit, John C. Driscoll, Xavier Gabaix, and David I. Laibson. 2009. "The Age of Reason: Financial Decisions over the Life-Cycle with Implications for Regulation." Brookings Papers on Economic Activity Fall (2009): $51-117$.

Bassett, William F., and Robin L. Lumsdaine. 1999. "Outlook, Outcomes, and Optimism.” Brown University. Unpublished manuscript.

Carstensen, Laura L. 2006. "The Influence of a Sense of Time on Human Development." Science 312:1913.

Crimmins, E. M., J. K. Kim, Kenneth M. Langa, and David Weir. 2011. "Assessment of Cognition Using Surveys and Neuropsychological Assessment: The Health and Retirement Study and the Aging, Demographics, and Memory Study." Journals of Gerontology Series B-Psychological Sciences and Social Sciences 66:162-71.

Fisher, Gwenith, Halimah Hassan, Willard L. Rodgers, and David R. Weir. 2012. "Health and Retirement Study - Imputation of Cognitive Functioning Measures: 1992 - 2010 Early Release." http://hrsonline.isr.umich.edu/modules/meta/xyear/ cogimp/desc/COGIMPdd.pdf.

Flynn, J. R. 1987. "Massive IQ Gains in 14 nations: What IQ Tests Really Measure." Psychological Bulletin 101:171-91.

Horn, J. L., and R. B. Cattell. 1967. "Age Differences in Fluid and Crystallized Intelligence." Acta Psychologica 26:107-29.

Horn, J. L., and J. J. McArdle. 2007. "Understanding Human Intelligence since Spearman." In Factor Analysis at 100. Historical Developments and Future Directions, edited by Robert Cudeck and Robert C. MacCallum, 206-44. Mahwah, NJ: LEA Publishers.

Hudomiet, P., and Robert J. Willis. 2013. "Estimating Second Order Probability Beliefs from Subjective Survival Data." Decision Analysis June (10): 152-70.

Hurd, Michael D. 2009. "Subjective Probabilities in Household Surveys." Annual Review of Economics 2009 (1): 543-62.

Hurd, Michael D., Paco Martorell, Adeline Delavande, Kathleen J. Mullen, and Kenneth M. Langa. 2013. "Monetary Costs of Dementia in the United States." New England Journal of Medicine 368 (14): 1326-34.

Hurd, Michael D., S. Rohwedder, and S. Winter. 2005. "Subjective Probabilities of Survival: An International Comparison." RAND. Unpublished manuscript.

Kézdi, G., and R. Willis. 2012. "Household Stock Market Beliefs and Learning." NBER Working Paper no. 17614, Cambridge, MA.

Manski, Charles F. 2004. "Measuring Expectations." Econometrica 72:1329-76.

McArdle, John J., E. Ferrer-Caja, F. Hamagami, and R. Woodcock. 2002. "Comparative Longitudinal Structural Analyses of the Growth and Decline of Multiple Intellectual Abilities over the Life Span.” Developmental Psychology 38 (1): $115-42$.

McArdle, John J., and Robert J. Willis. 2011. "Cognitive Aging and Human Capital." In Grounding Social Sciences in Cognitive Sciences, edited by Ron Sun, 354-84. Cambridge: MIT Press.

Plassman, B., K. Langa, G. Fisher, S. Heeringa, D. Weir, M. Ofstedal, J. Burke et al. 2007. "Prevalence of Dementia in the United States: The Aging, Demographics, and Memory Study." Neuroepidemiology 29 (1-2): 125-32.

Reuter-Lorenz, P. A., and D. C. Park. 2010. "Human Neuroscience and the Aging Mind: A New Look at Old Problems." Journal of Gerontology: Psychological Sciences 65B (4): 405-15. 